\title{
Promoter methylation of Wrap53 $\alpha$, an antisense transcript of p53, is associated with the poor prognosis of patients with non-small cell lung cancer
}

\author{
DONG SUN KIM ${ }^{1}$, WON KEE LEE ${ }^{2}$ and JAE YONG PARK ${ }^{3}$ \\ ${ }^{1}$ Department of Anatomy, BK21 Plus KNU Biomedical Convergence Program; Departments of ${ }^{2}$ Preventive Medicine \\ and ${ }^{3}$ Internal Medicine, School of Medicine, Kyungpook National University, Daegu 702-422, Republic of Korea
}

Received May 11, 2018; Accepted August 30, 2018

DOI: $10.3892 / \mathrm{ol} .2018 .9404$

\begin{abstract}
Lung cancer, of which non-small cell lung cancer (NSCLC) accounts for $\sim 85 \%$ of cases, remains a leading cause of cancer-associated mortality and morbidity worldwide. Tumor suppressor p53 is a master regulator of diverse cellular processes and is a therapeutic target in cancer. However, many aspects of its transcriptional regulation are still not well defined. WD repeat containing antisense to TP53 $\alpha$ (Wrap53 $\alpha$ ) a newly identified natural antisense transcript of p53, can regulate p53 expression following DNA damage. The present study determined the methylation status of the Wrap $53 \alpha$ promoter in primary lung tissues using methylation-specific polymerase chain reaction and evaluated its associations with clinicopathological features and survival in patients with NSCLC. The Wrap53 $\alpha$ promoter was methylated in $12(8.2 \%)$ of 146 malignant tissues. Its methylation was associated with the downregulation of its transcription and was frequently detected in patients with stages II-IIIA $(\mathrm{P}=0.03)$, and p53 mutation-negative cases $(\mathrm{P}=0.08)$. Methylation of Wrap $53 \alpha$ promoter was associated with worse overall survival of total patients with a borderline significance [adjusted Hazard Ratio $(\mathrm{HR})=2.44,95 \%$ Confidence Interval $(\mathrm{CI})=0.98-6.04, \mathrm{P}=0.05]$. Notably, Wrap53 $\alpha$ promoter methylation significantly associated with poor overall survival in p53 mutation-negative patients ( $\log$-rank $\mathrm{P}=0.01$, adjusted $\mathrm{HR}=2.92,95 \% \mathrm{CI}=1.00-8.60, \mathrm{P}=0.05)$, but not in patients with p53 mutations. The results of the present study suggest that Wrap53 $\alpha$ may serve a role in the pathogenesis of a subset of lung cancer, and its methylation may be considered to be a prognostic marker for surgically resected NSCLC patients.
\end{abstract}

Correspondence to: Professor Jae Yong Park, Department of Internal Medicine, School of Medicine, Kyungpook National University, 2-101 Dongin-dong, Jung-gu, Daegu 702-422, Republic of Korea

E-mail: jaeyong@knu.ac.kr

Key words: DNA methylation, non-small cell lung cancer, prognosis, p53, WD repeat containing antisense to TP53 $\alpha$
However, further studies with a larger sample size are required to confirm this finding.

\section{Introduction}

Lung cancer is the leading cause of cancer-related mortality in many countries, called 'a global scourge' with a dismal prognosis. Diagnosis is frequently made at an advanced stage when prognosis is poor and therapeutic options are limited (1). The molecular mechanisms underlying global variations in lung cancer biology remains poorly understood (2). Therefore, it is necessary to identify multiple biomarkers for early detection and prognosis. Transcriptional silencing of genes by $\mathrm{CpG}$ islands (CGIs) methylation is now recognized as a crucial component in lung cancer initiation and progression (3). In addition, gene-specific hyper-methylation has emerged as an important factor in the earliest stages of preinvasive lung cancer related to tobacco smoking, a major etiological factor (4).

Tumor suppressor p53 is a cellular gatekeeper that guards against genetic instability and abnormality by sensing multiple stress signals, including DNA damage, oncogene activation, and hypoxia (5). Lung cancer has a higher p53 mutation rate compared to other kinds of cancer (6). Expression of p53 is tightly controlled through multiple regulatory layers, but limited information is available on how p53 is transcriptional and epigenetically regulated (7). Recently, WD40 repeat containing antisense to p53 (Wrap53) (also known as WDR79/TCAB1) was found to be a natural antisense transcript (NAT) of p53 that regulates endogenous p53 mRNA levels by targeting the 5'untranslated region (UTR) (8). Transcripts initiated from exon $1 \alpha, 1 \beta$, and $1 \gamma$ are called Wrap $53 \alpha$, Wrap $53 \beta$, and Wrap $53 \gamma$, respectively. Exon $1 \alpha$ directly overlaps the first exon of p53 in an antisense fashion and forms an RNA-RNA hybrid with $p 53$ mRNA to protect it from degradation (8). Interestingly, Wrap $53 \alpha$ transcript is upregulated by cancer therapeutic drugs and miR-4732-5p has a binding site in the 5' UTR of the Wrap53 $\alpha$ transcript (9-11). However, overexpression of the WRAP53 protein, mainly produced from the Wrap53 $\beta$ transcript, is linked to progression of several types of tumors, including lung cancer (12-15). Importantly, neither 
$\beta$ - or $\gamma$-transcripts, nor WRAP53 protein, has any effects on p53 when overexpressed or knocked down. Therefore, it is conceivable that dysfunction of Wrap $53 \alpha$ could contribute to tumorigenesis by failing to sustain $p 53$ expression and function in wild-type (WT) p53-carrying tumors. In order to test this hypothesis and understand the biological role of Wrap $53 \alpha$ in lung cancer, we investigated the methylation status of the Wrap $53 \alpha$ promoter in resected primary non-small cell lung cancers (NSCLC) using methylation-specific polymerase chain reaction (MSP) and assessed the correlation of these results with clinicopathological characteristics.

\section{Materials and methods}

Patients and tissue samples. Tumor and corresponding non-malignant lung tissue specimens $(n=146)$ were provided by the National Biobank of Korea, Kyungpook National University Hospital (KNUH; Daegu, Korea), which is supported by the Ministry of Health, Welfare, and Family Affairs. The present study was conducted with the approval of the Ethics Committee of KNUH (no. 2014-04-210) and written informed consent was obtained from all of the participants prior to obtaining the samples. The clinicopathological characteristics of the patients are summarized in Table I.

Genomic DNA isolation and methylation analysis. Genomic DNA was extracted using a QIAamp DNA Mini kit (QIAGEN, Valencia, CA). After treatment of the genomic DNA with sodium bisulfite, the methylation status of Wrap53 $\alpha$ promoter encompassing the transcription start site was analyzed using MSP with primers specific for either unmethylated or methylated alleles. The primer sequences for Wrap $53 \alpha$ were described in Table II. All polymerase chain reaction (PCR) amplifications were carried out using reagents supplied in a GeneAmp DNA Amplification kit with AmpliTaq Gold as the polymerase (PE Applied Biosystems, Foster City, CA, USA) on a PTC-100 thermal cycler (MJ Research, Watertown, MA, USA). CpGenome ${ }^{\mathrm{TM}}$ Universal methylated and unmethylated DNA (Chemicon, Temecula, CA, USA) was used as a positive control for the methylated and unmethylated genes, respectively. Negative control samples without DNA were included for each set of PCR. PCR products were analyzed on $2 \%$ agarose gel, stained with ethidium bromide, and visualized under UV light. Each MSP was repeated at least once to confirm the results.

Cell culture, total RNA isolation, and semi-quantitative (sq)-PCR. Ten human NSCLC cell lines (A549, HCC827, H23, H358, H520, H522, H1299, H1703, H2009 and PC9) were obtained from the American Type Culture Collection (ATCC, Manassas, VA). All cells were propagated according to instructions from the ATCC. HCC 827 cells were treated with $20 \mu \mathrm{M} 5$-AzadC for 3 days and the culture media was changed daily. Total RNA was extracted from cultured cells and primary tumor tissues using TRIzol (Invitrogen; Thermo Fisher Scientific, Inc., Waltham, MA, USA). After removal of residual DNA, first-strand cDNA was synthesized from total RNA using SuperScript preamplification (Invitrogen; Thermo Fisher Scientific, Inc.) according to the manufacturer's instructions. The resulting cDNA was amplified with Wrap53 $\alpha$ - and
Table I. Correlation between Wrap $53 \alpha$ promoter methylation status and characteristics of non-small cell lung cancer patients.

\begin{tabular}{|c|c|c|}
\hline Variables & Methylation, n (\%) & P-value \\
\hline All subjects $(n=146)$ & $12(8.2)$ & \\
\hline \multicolumn{3}{|l|}{ Age (years) } \\
\hline$\leq 64(n=76)$ & $7(9.2)$ & 0.66 \\
\hline$>64(\mathrm{n}=70)$ & $5(7.1)$ & \\
\hline \multicolumn{3}{|l|}{ Sex } \\
\hline Men $(n=98)$ & $8(8.2)$ & 0.97 \\
\hline Women $(n=48)$ & $4(8.3)$ & \\
\hline \multicolumn{3}{|l|}{ Smoking status } \\
\hline Ever $(n=101)$ & $8(7.9)$ & 0.84 \\
\hline Never $(n=45)$ & $4(8.9)$ & \\
\hline \multicolumn{3}{|l|}{ Histological types } \\
\hline SQC $(n=43)$ & $2(4.7)$ & 0.31 \\
\hline $\operatorname{ADC}(n=103)$ & $10(9.7)$ & \\
\hline \multicolumn{3}{|l|}{ Pathologic stage } \\
\hline Stage I $(\mathrm{n}=91)$ & $4(4.4)$ & 0.03 \\
\hline Stage II-IIIA ( $\mathrm{n}=55)$ & 8 (14.6) & \\
\hline \multicolumn{3}{|l|}{ p53 mutations } \\
\hline Negative $(n=87)$ & $10(11.5)$ & 0.08 \\
\hline Positive $(n=59)$ & $2(3.4)$ & \\
\hline
\end{tabular}

SQC, squamous cell carcinoma; ADC, adenocarcinoma; Wrap53 $\alpha$, WD repeat containing antisense to TP53 $\alpha$.

p53-specific primers as previously described (8). The following thermocycling conditions were applied: $95^{\circ} \mathrm{C}$ for $2 \mathrm{~min}$, then 30 cycles of $95^{\circ} \mathrm{C}$ for $1 \mathrm{~min}, 58^{\circ} \mathrm{C}$ for $1 \mathrm{~min}$ and $72^{\circ} \mathrm{C}$ for $1 \mathrm{~min}$, and a final extension at $72^{\circ} \mathrm{C}$ for $10 \mathrm{~min}$. Amplification of $G A P D H$ was used as an internal loading control. All primer sequences were described in Table II.

Mutational analysis of p53 gene. P53 mutational analysis of the entire coding regions (exons 2-11), including exon/intron boundaries, was performed by PCR-based direct sequencing. The primers and conditions for PCR reactions were described previously (16). Sequencing was done using an ABI Prism 3100 Genetic Analyzer (PE Applied Biosystems). All sequence variants were confirmed by sequencing the products of independent PCR amplifications in both directions.

Statistical analysis. The associations between methylation status and clinicopathological characteristics were analyzed using a chi-square test for categorical variables. Logistic regression analysis was conducted to estimate the association between methylation status and the covariates of age, sex, exposure to tobacco smoke, and histology. The overall survival (OS) of NSCLC patients according to methylation status of the Wrap $53 \alpha$ promoter was compared using the Kaplan-Meier method and the log-rank test. Hazard ratios (HRs) and 95\% confidence intervals (CIs) were estimated using a multivariate Cox proportional hazard model. Data were analyzed using SAS v9.4 software (SAS Institute, Inc., Cary, NC, USA). 
Table II. Primer sequences used for MSP and sqPCR.

\begin{tabular}{|c|c|c|}
\hline Primer & Forward primer ( $5^{\prime}$ to $\left.3^{\prime}\right)$ & Reverse primer ( $5^{\prime}$ to $\left.3^{\prime}\right)$ \\
\hline \multicolumn{3}{|l|}{ MSP } \\
\hline U-MSP & AATATATGGAGTTGAGAGTTT & AAAAACATACTTTCCACAACA \\
\hline M-MSP & AATATACGGAGTCGAGAGTTC & AAAAACGTACTTTCCACGACG \\
\hline \multicolumn{3}{|l|}{ sqPCR } \\
\hline Wrap $53 \alpha$ & CGGAGCCCAGCAGCTACC & TTGTGCCAGGAGCCTCGCA \\
\hline Wrap $53 \beta$ & GTCCCGGCTCCGCGGGTTC & GGCTGAGGACATCAGAGAATACCAGC \\
\hline P53 & GACGGTGACACGCTTCCCTGGAT & CGTGCAAGTCACAGACTTGGCTGTC \\
\hline GAPDH & CATGACAACTTTGGTATCGTG & GTGTCGCTGTTGAAGTCAGA \\
\hline
\end{tabular}

MSP, methylation-specific polymerase chain reaction; sqPCR, semi-quantitative polymerase chain reaction; M-MSP, MSP for the methylated allele; U-MSP, MSP for the unmethylated allele.

A MSP

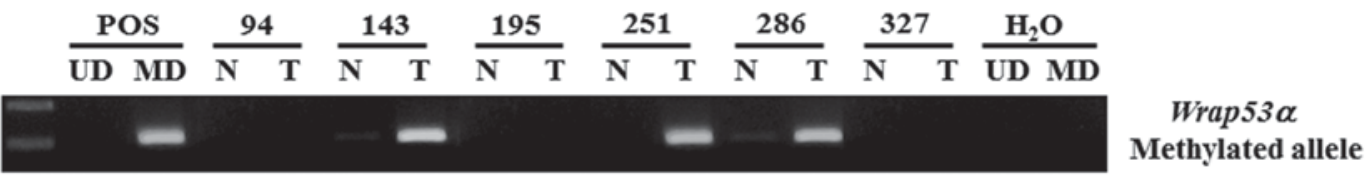

B sqPCR

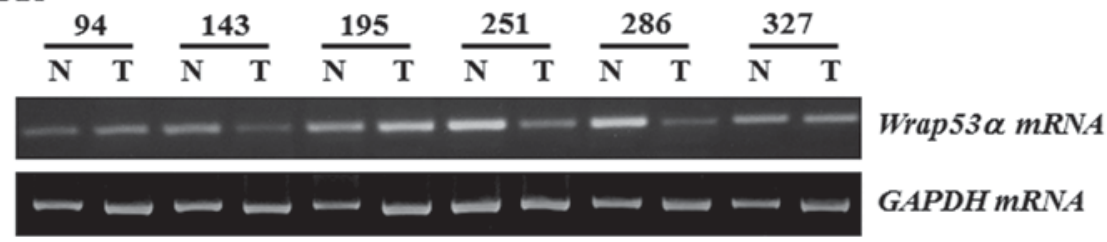

Figure 1. Representative results of MSP and sqPCR analysis of Wrap53a gene in NSCLC patients. (A) The methylation status of the Wrap53 $\alpha$ promoter in NSCLCs was analyzed by MSP. CpGenome ${ }^{\mathrm{TM}}$ Universal MD or UD was used as a POS for the methylated or unmethylated products, respectively. Water was used as a negative control. (B) Expression of Wrap53 $\alpha$ mRNA was measured in primary tissues from NSCLC patients by sqPCR. Amplified products were run on $2 \%$ agarose gel and appeared at positions corresponding to the expected base pair lengths. Amplification of GAPDH was used as an internal loading control. sqPCR, semi-quantitative polymerase chain reaction; N, non-malignant tissue; T, tumor tissues; M-MSP, amplified product with primers that recognize the

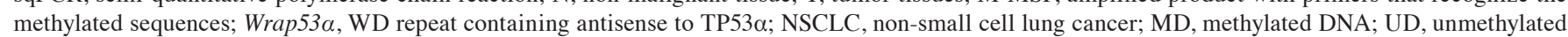
DNA; POS, positive control.

$\mathrm{P}<0.05$ was considered to indicate a statistically significant difference.

\section{Results}

Methylation status and expression of Wrap53a gene in NSCLC samples. We have analyzed the methylation status of the human Wrap53 $\alpha$ gene in 146 primary NSCLCs and their corresponding nonmalignant lung tissues using MSP. There were no classical CGIs in the 5'-flanking region of the human Wrap53 $\alpha$ gene, including the first exon, but 13 CGIs were found from -150 to +30 bp upstream of the transcription start site. Thus, we designed the MSP primer pairs to cover this region. Methylated alleles of representative samples were shown as in Fig. 1A. Unmethylated bands were detected in most of the nonmalignant and malignant tissues (data not shown), thus confirming the integrity of the DNA in those samples. Bisulfite-sequencing of the representative PCR products confirmed the assigned methylation status and showed that all cytosines at non-CpG sites were converted to thymine (data not shown), ruling out the possibility of incomplete bisulfite conversion. Wrap $53 \alpha$ methylation was exclusively detected in malignant tissues at a frequency of $8.2 \%(12 / 146)$, suggesting that Wrap $53 \alpha$ promoter methylation may be a tumor-associated event during NSCLC tumorigenesis.

To determine whether $\mathrm{CpG}$ methylation was involved in the regulation of Wrap53 $\alpha$ expression, we analyzed Wrap $53 \alpha$ mRNA levels in representative tissue specimens. sqPCR analysis showed low or undetectable levels of Wrap53a transcripts in tumor tissues with a methylated allele, whereas high levels were detected in tumor and non-tumor lung tissues with an unmethylated allele (Fig. 1B). We have further confirmed these results in 10 human NSCLC cell lines. Comparison of methylation status with sqPCR findings demonstrated that Wrap $53 \alpha$ mRNA was present in all examined cell lines except a HCC827 cell line that had methylated alleles (Fig. 2A). After treatment with the demethylating agent 5-AzadC for 3 days, HCC827 cells exhibited the disappearance of methylated alleles and induced the re-expression of Wrap $53 \alpha$ mRNA, resulting in increased p53 mRNA levels (Fig. 2B). These 


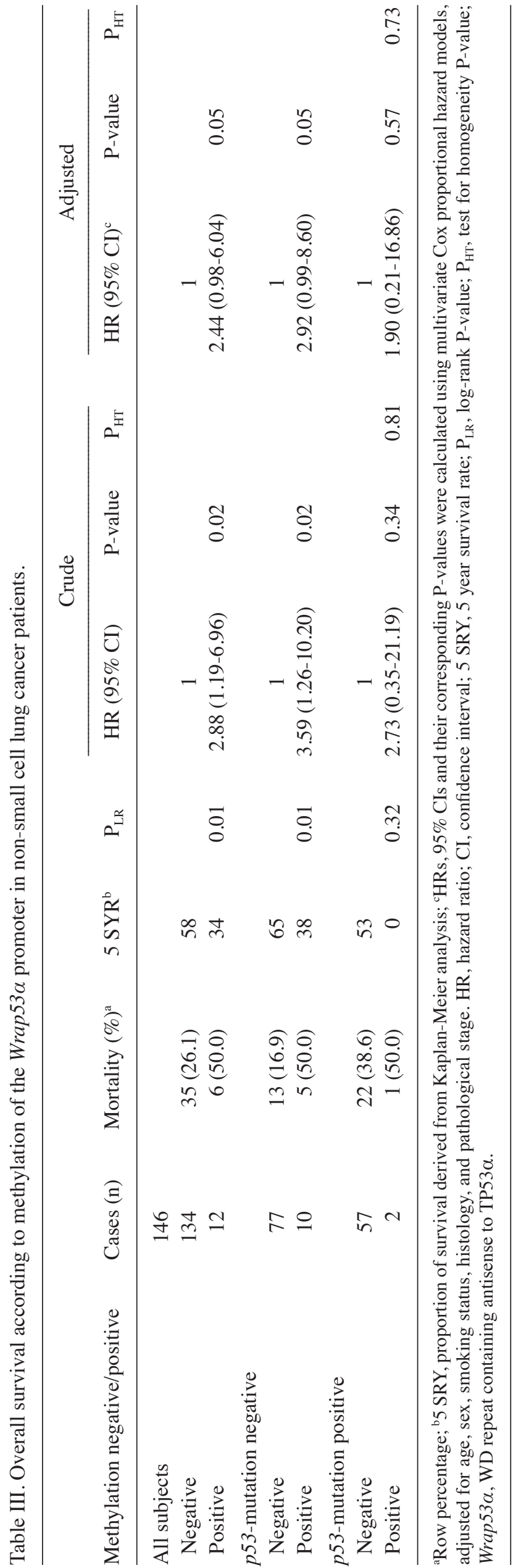

A MSP and sqPCR

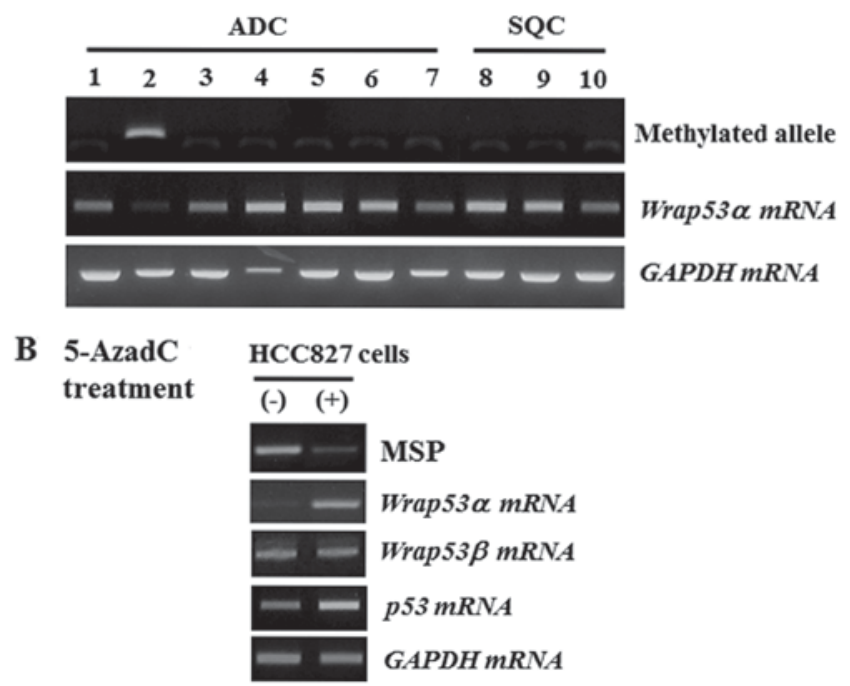

Figure 2. MSP and sqPCR analysis of Wrap53a gene in NSCLC cell lines. (A) The methylation status of the Wrap53a promoter was analyzed in 10 cell lines by MSP. Expression of Wrap $53 \alpha$ mRNA was performed on the same cell lines by sqPCR. Lane 1, A549; Lane 2, HCC827; Lane 3, H23; Lane 4, H358; Lane 5, H522; Lane 6, H1299; Lane 7, PC9; Lane 8, H520; Lane 9, H1703; Lane 10, H2009. Lanes 1-7, ADC; Lanes 8-10, SQC. (B) Methylation status and expression of Wrap53 $\alpha$ was analyzed in HCC827 cells following $20 \mu \mathrm{M}$ 5-AzadC treatment for 3 days. Simultaneously, Wrap53a and p53 mRNA levels were measured. GAPDH was amplified as an internal loading control. $(-)$, vehicle alone; (+), 5-AzadC addition; MSP, methylation-specific polymerase chain reaction; sqPCR, semi-quantitative polymerase chain reaction; Wrap53 $\alpha$, WD repeat containing antisense to TP53 $\alpha$; NSCLC, non-small cell lung cancer; ADC, adenocarcinoma; SQC, squamous cell carcinoma.

results suggest that $\mathrm{CpG}$ island methylation may be a mechanism for downregulating the expression of Wrap $53 \alpha$ gene.

Association of Wrap53a promoter methylation with clinicopathological parameters and clinical outcomes. Wrap $53 \alpha$ promoter methylation was significantly more frequent in stages II-IIIA tumors than stages I tumors $(\mathrm{P}=0.03)$ (Table I). In addition, its methylation was more frequently detected in $p 53$ mutation-negative cases than in p53 mutation-positive cases with borderline significance $(\mathrm{P}=0.08)$. However, no significant correlation was observed between its methylation and any other factors, such as age, sex, or smoking status (Table I). Next, Kaplan-Meier survival analysis was carried out to determine the prognostic potential of Wrap $53 \alpha$ promoter methylation. Interestingly, the patients with the methylation had worse OS compared to those without Wrap53 $\alpha$ methylation [log-rank $\left.P\left(P_{\mathrm{L}-\mathrm{R}}\right)=0.01\right]$ (Table III and Fig. 3). When stratified according to clinicopathological characteristics of patients, Wrap53 promoter methylation was significantly associated with an unfavorable survival in a subset of patients including younger, female, never-smoker, squamous cell carcinoma, and $p 53$ mutation-negative $\left(P_{\mathrm{L}-\mathrm{R}}=0.0003\right.$, $0.03,0.02,0.01$, and 0.01 , respectively) (data not shown). To evaluate the Wrap53 $\alpha$ promoter methylation as an independent prognostic factor in NSCLC, we further analyzed the data using the Cox proportional hazards regression adjusting for possible confounders of survival. Methylation of Wrap53a promoter was significantly associated with worse OS of total patients [adjusted HR $\left({ }_{\text {adj }} \mathrm{HR}\right)=2.44,95 \% \mathrm{CI}=0.98-6.04$, 

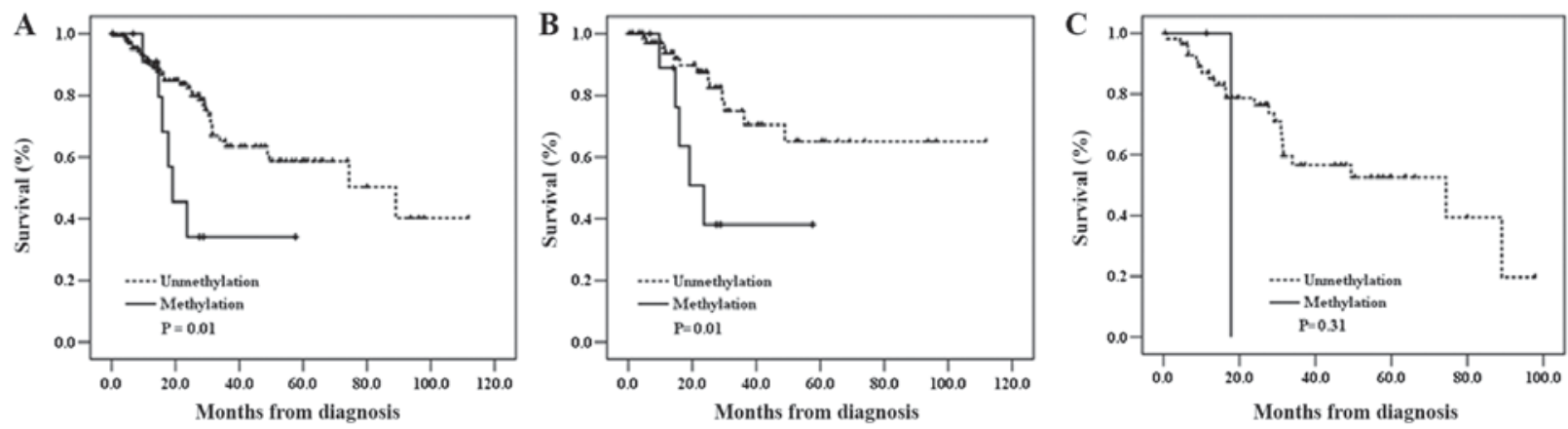

Figure 3. Kaplan-Meier survival curves of non-small cell lung cancer patients according to Wrap53a promoter methylation status. (A) Overall patients, (B) patients without $p 53$ mutations and (C) patients with $p 53$ mutations. P-values were calculated via a log-rank test. Wrap $53 \alpha$, WD repeat containing antisense to TP53 $\alpha$.

$\mathrm{P}=0.05]$. Notably, Wrap53 $\alpha$ promoter methylation significantly associated with poor OS in $p 53$ mutation-negative patients $\left(_{\text {adj }} \mathrm{HR}=2.92,95 \% \mathrm{CI}=1.00-8.60, \mathrm{P}=0.05\right.$; Table III) but not in patients with p53 mutations. Moreover, Wrap53 $\alpha$ promoter methylation exhibited a trend toward worse OS in patients with stages II-IIA $\left({ }_{\text {adj }} \mathrm{HR}=2.76,95 \% \mathrm{CI}=0.93-8.22, \mathrm{P}=0.07\right)$ (data not shown). These results suggest that Wrap53 $\alpha$ may play an important role in lung cancer pathogenesis and its methylation could be considered as a prognostic marker for NSCLC patients.

\section{Discussion}

Although the majority of investigations concerned with P53 have focused on coding regions, recent studies have highlighted the significant roles that regulatory elements located in p53 mRNA play, particularly the 5'UTR that displays high conservation and immutability $(17,18)$. Wrap53 antisense RNA targets p53 mRNA via the 5'UTR and increases p53 protein levels, indicating that dysfunction of Wrap53 itself may be a separate cause of cancer. Wrap53 has three different start exons: Exon $1 \alpha, 1 \beta$, and $1 \gamma$. Exon $1 \alpha$ and $1 \gamma$ match the first exon and intron, respectively, of p53 in a cis-antisense manner. Exon $1 \beta$ does not produce transcripts that are complementary to any section of p53 (8). Moreover, knockdown of Wrap53 $\alpha$ reduces $p 53$ abundance (8). There are several studies focusing on the function and expression of Wrap53 $\beta$ transcript in tumor progression (12-15), however, the exact function of have no information about is available. Thus, we focused on the methylation status of the Wrap $53 \alpha$ promoter. The discovery of Wrap $53 \alpha$ would elucidate the role of NAT-mediated gene regulation in the P53 pathway. NATs, as a member of long non-coding RNAs, occur ubiquitously in mammals and are crucial players in carcinogenesis, invasion, and metastasis (19). These RNAs regulate gene expression through direct interaction with sense transcripts or indirect interactions with other targets, such as DNA methyltransferases (DNMTs), histone acetylases and histone deacetylases. Many NATs interact with cancer relevant genes such as $p 53, p 15$, p21, RB1, and PTEN (20). Taken together, our findings provide new insights that NATs could be a potential rich sources of biomarkers for use in diagnosis and prognosis of cancer.

Although the molecular mechanisms contributing to promoter methylation of Wrap53 $\alpha$ remain elusive, there is evidence that malignant transformation associated with chronic inflammation, persistent viral infection, cigarette smoking, and oxidative stress can upregulate the expression and activity of DNMTs through transcriptional and post-translational regulation (21-24). Interestingly, Lin et al (25) have shown that dysregulation of p53 control leads to DNMT1 and DNMT3A overexpression, resulting in promoter hypermethylation of multiple tumor suppressor genes in NSCLC patients (26). Thus, it is likely that the overexpressed DNMT can induce Wrap $53 \alpha$ hypermethylation.

It is noteworthy that Wrap $53 \alpha$ methylation was significantly associated with unfavorable survival in a subset of NSCLC patients, especially for $p 53$ mutation-negative patients. The downregulation of Wrap $53 \alpha$ transcripts by promoter methylation could destabilize $p 53$ mRNA to reduce tumor suppressor activity of the WT P53, contributing to poor prognosis. Smoking causes a high percentage of missense mutations in the DNA-binding domain of $p 53$, producing a striking gain-of function phenotype (6). In addition, mutant P53 can drive cancer by subverting multiple tumor suppression pathways independent of WT p53 (27). Mutations of the p53 gene usually but not always lead to an increased half life of the $p 53$ protein, and result in a nuclear accumulation of protein (27). Consequently, p53 alteration that can be detected as either protein overexpression or mutation makes the problem more complicated. Alternatively, alterations in regulators of P53 provide an alternative way to deregulate P53 function in p53 WT tumors, but are likely redundant in tumors that already have a dysfunctional P53 protein. Indeed, we found that Wrap $53 \alpha$ methylation showed a tendency for p53 mutation-negative patients, indicating the absence of concomitant alterations in $p 53$ and Wrap53a. Mutual exclusive alterations is frequently observed in cancer, being believed to occur between functionally related genes (28). Taken together, it could be speculated that the effect of Wrap $53 \alpha$ promoter methylation on the clinical outcome could be more noticeable in patients with WT P53. Therefore, our findings suggest that the close interplay between the Wrap53 $\alpha$ and $p 53$ might be involved in the pathogenesis of NSCLC. However, further studies with larger sample sizes are required to establish that Wrap $53 \alpha$ methylation is a useful prognostic indicator for patients with NSCLC.

The present study has shown that the Wrap $53 \alpha$ promoter was methylated in a subset of NSCLCs, and its methylation was significantly associated with unfavorable OS of patients, particularly in patients with p53 mutation negative, suggesting that Wrap53 $\alpha$ methylation status could be informative for 
prediction of NSCLC prognosis. Although the current study did not have a full viewpoint because of the small sample size and no information regarding P53 protein expression, it is the first report to demonstrate an aberrant methylation of the Wrap $53 \alpha$ promoter in NSCLC and may provide new pieces in the P53 targeting puzzle for cancer therapy.

\section{Acknowledgements}

Not applicable.

\section{Funding}

The present study was supported by a grant from the Korea Health Technology R\&D Project through the Korea Health Industry Development Institute (KHIDI), funded by the Ministry of Health and Welfare (grant no. HI4C0402).

\section{Availability of data and materials}

The analyzed datasets generated during the study are available from the corresponding author on reasonable request.

\section{Authors' contributions}

DSK contributed to the experimental design and implementation, performed the experiments and data analysis, and drafted the manuscript. WKL performed the statistical analyses. JYP contributed to experimental implementation, interpreted the patient data and modified the manuscript. All authors have read and approved the final manuscript.

\section{Ethics approval and consent to participate}

The present study was approved by the Ethics Committee of KNUH (no. 2014-04-210). Written informed consent was obtained from all participants or their families prior to obtaining the samples.

\section{Patient consent for publication}

All participants provided written informed consent for the publication of any associated data and accompanying images.

\section{Competing interests}

The authors declare that they have no competing interests.

\section{References}

1. Torre LA, Bray F, Siegel RL, Ferlay J, Lortet-Tieulent J and Jemal A: Global cancer statistics, 2012. CA Cancer J Clin 65 87-108, 2015.

2. McIntyre A and Ganti AK: Lung cancer-A global perspective. J Surg Oncol 115: 550-554, 2017.

3. Kerr KM, Galler JS, Hagen JA, Laird PW and Laird-Offringa IA: The role of DNA methylation in the development and progression of lung adenocarcinoma. Dis Markers 23: 5-30, 2007.

4. Brzeziańska E, Dutkowska A and Antczak A: The significance of epigenetic alterations in lung carcinogenesis. Mol Biol Rep 40: 309-325, 2013.

5. Green DR and Kroemer G: Cytoplasmic functions of the tumour suppressor p53. Nature 458: 1127-1130, 2009.
6. Gibbons DL, Byers LA and Kurie JM: Smoking, p53 mutation, and lung cancer. Mol Cancer Res 12: 3-13, 2014.

7. Saldaña-Meyer R and Recillas-Targa F: Transcriptional and epigenetic regulation of the p53 tumor suppressor gene. Epigenetics 6: 1068-1077, 2011.

8. Mahmoudi S, Henriksson S, Corcoran M, Méndez-Vidal C, Wiman KG and Farnebo M: Wrap53, a natural p53 antisense transcript required for p53 induction upon DNA damage. Mol Cell 33: 462-471, 2009.

9. Bug M and Dobbelstein M: Anthracyclines induce the accumulation of mutant p53 through E2F1-dependent and -independent mechanism. Oncogene 30: 3612-3624, 2011.

10. Yuan JM, Li XD, Liu ZY, Hou GQ, Kang JH, Huang DY and Du SX: Cisplatin induces apoptosis via upregulating Wrap53 in U-2OS osteosarcoma cells. Asian Pac J Cancer Prev 12: 3465-3469, 2011.

11. Pouladi N, Kouhsari SM, Feizi MH, Gavgani RR and Azarfam P: Overlapping region of p53/wrap53 transcripts: Mutational analysis and sequence similarity with microRNA-4732-5p. Asian Pac J Cancer Prev 14: 3503-3507, 2013.

12. Mahmoudi S, Henriksson S, Farnebo L, Roberg K and Farnebo M: WRAP53 promotes cancer cell survival and is a potential target for cancer therapy. Cell Death Dis 2: e114, 2011.

13. Zhang H, Wang DW, Adell G and Sun XF: WRAP53 is an independent prognostic factor in rectal cancer-a study of Swedish clinical trial of preoperative radiotherapy in rectal cancer patients. BMC Cancer 12: 294, 2012.

14. Rao X, Huang D, Sui X, Liu G, Song X, Xie J and Huang D: Overexpression of WRAP53 is associated with development and progression of esophageal squamous cell carcinoma. PLoS One 9: e91670, 2014.

15. Yuan XS, Cao LX, Hu YJ, Bao FC, Wang ZT, Cao JL, Yuan P, Lv W and $\mathrm{Hu}$ J: Clinical, cellular, and bioinformatic analyses reveal involvement of WRAP53 overexpression in carcinogenesis of lung adenocarcinoma. Tumor Biol 39: 1010428317694309, 2017.

16. Lee EB, Jin G, Lee SY, Park JY, Kim MJ, Choi JE, Jeon HS, Cha SI, Cho S, Kim CH, et al: TP53 mutations in Korean patients with non-small cell lung cancer. J Korean Med Sci 25: 698-705, 2010.

17. Swiatkowska A, Zydowicz P, Sroka J and Ciesiołka J: The role of the $5^{\prime}$ terminal region of p53 mRNA in the p53 gene expression. Acta Biochim Pol 63: 645-651, 2016.

18. Alonso S, Izarirre N, López S, Smith-Zubiaga I, Hervella M, Boyano MD, Arroyo-Berdugo Y, Gardeazabal J, Díaz-Ramón JL, Sánchez Díez A, et al: The diversity profile of TP53 is influenced by positive selection on the immediately upstream locus WDR79. Hum Hered 69: 34-44, 2010.

19. Nie L, Wu HJ, Hsu JM, Chang SS, Labaff AM, Li CW, Wang Y, Hsu JL and Hung MC: Long non-coding RNAs: Versatile master regulators of gene expression and crucial players in cancer. Am J Transl Res 4: 127-150, 2012.

20. Su WY, Xing H and Fang JY: Natural antisense transcripts regulate gene expression in an epigenetic manner. Biochem Biophys Res Commun 396: 177-181, 2010.

21. Kanai Y: Alterations of DNA methylation and clinicopathological diversity of human cancers. Pathol Int 58: 544-558, 2008.

22. Wu Q and Ni X: ROS-mediated DNA methylation pattern alterations in carcinogenesis. Curr Drug Targets 16: 13-19, 2015.

23. Lin RK and Wang YC: Dysregulated transcriptional and post-translational control of DNA methyltransferases in cancer. Cell Biosci 4: 46, 2014.

24. Jin T,Hao J and Fan D: Nicotine induces aberrant hypermethylation of tumor suppressor genes in pancreatic epithelial ductal cells. Biochem Biophys Res Commun 499: 934-940, 2018.

25. Lin RK, Wu CY, Chang JW, Juan LJ, Hsu HS, Chen CY, Lu YY, Tang YA, Yang YC, Yang PC and Wang YC: Dysregulation of p53/Sp1 control leads to DNA methyltransferase-1 overexpression in lung cancer. Cancer Res 70: 5807-5817, 2010.

26. Tang YA, Tsai YT, Lin RK, Hsu HS, Chen CY and Wang YC: Dysregulation of p53 and RB transcriptional control leads to overexperssion of DNA methyltransferase in lung cancer. J Cancer Res Pract 1: 14-27, 2014.

27. Yue X, Zhao Y, Xu Y, Zheng M, Feng Z and Hu W: Mutant p53 in cancer: Accumulation, gain-of-function, and therapy. J Mol Biol 429: 1595-1606, 2017.

28. Yeang $\mathrm{CH}, \mathrm{McC}$ Comick $\mathrm{F}$ and Levine A: Combinatorial patterns of somatic gene mutations in cancer. FASEB J 22: 2605-2622, 2008.

This work is licensed under a Creative Commons Attribution-NonCommercial-NoDerivatives 4.0 International (CC BY-NC-ND 4.0) License. 